\title{
Accurate Ground-State Energies of Solids and Molecules from Time-Dependent Density-Functional Theory
}

\author{
Olsen, Thomas; Thygesen, Kristian Sommer
}

Published in:

Physical Review Letters

Link to article, DOI:

10.1103/PhysRevLett.112.203001

Publication date:

2014

Document Version

Publisher's PDF, also known as Version of record

Link back to DTU Orbit

Citation (APA):

Olsen, T., \& Thygesen, K. S. (2014). Accurate Ground-State Energies of Solids and Molecules from TimeDependent Density-Functional Theory. Physical Review Letters, 112(20), 203001.

https://doi.org/10.1103/PhysRevLett.112.203001

\section{General rights}

Copyright and moral rights for the publications made accessible in the public portal are retained by the authors and/or other copyright owners and it is a condition of accessing publications that users recognise and abide by the legal requirements associated with these rights.

- Users may download and print one copy of any publication from the public portal for the purpose of private study or research.

- You may not further distribute the material or use it for any profit-making activity or commercial gain

- You may freely distribute the URL identifying the publication in the public portal 


\title{
Accurate Ground-State Energies of Solids and Molecules from Time-Dependent Density-Functional Theory
}

\author{
Thomas Olsen* and Kristian S. Thygesen \\ Center for Atomic-Scale Materials Design (CAMD) and Center for Nanostructured Graphene (CNG), \\ Department of Physics, Technical University of Denmark, DK-2800 Kongens Lyngby, Denmark
}

(Received 6 December 2013; published 21 May 2014)

\begin{abstract}
We demonstrate that ground-state energies approaching chemical accuracy can be obtained by combining the adiabatic-connection fluctuation-dissipation theorem with time-dependent densityfunctional theory. The key ingredient is a renormalization scheme, which eliminates the divergence of the correlation hole characteristic of any local kernel. This new class of renormalized kernels gives a significantly better description of the short-range correlations in covalent bonds compared to the random phase approximation (RPA) and yields a fourfold improvement of RPA binding energies in both molecules and solids. We also consider examples of barrier heights in chemical reactions, molecular adsorption, and graphene interacting with metal surfaces, which are three examples where the RPA has been successful. In these cases, the renormalized kernel provides results that are of equal quality or even slightly better than the RPA, with a similar computational cost.
\end{abstract}

DOI: 10.1103/PhysRevLett.112.203001

PACS numbers: 31.15.E-, 31.15.ve, 31.15.vn, 71.15.Mb

The calculation of ground-state energies with a precision on the order of $k_{B} T$ at room temperature (around $1 \mathrm{kcal} / \mathrm{mol}$ or $43 \mathrm{meV}$ ) is a long-standing challenge in computational chemistry and materials science. This degree of precision, sometimes referred to as "chemical accuracy," can in principle be achieved using quantum chemistry wave function methods [1]. However, the extreme scaling of such methods limits their usage to relatively small systems, and their application to periodic systems in general and metals in particular, seems highly nontrivial $[2,3]$.

Recently, the adiabatic-connection fluctuation-dissipation theorem (ACFDT) has become a popular method for obtaining electronic ground-state energies from first principles. With this approach the electronic correlation energy is obtained from an approximation to the dynamic density response function $\chi^{\lambda}(\omega)$, which can be obtained from timedependent density-functional theory (TDDFT) through the Dyson equation

$$
\chi^{\lambda}(\omega)=\chi^{\mathrm{KS}}(\omega)+\chi^{\mathrm{KS}}(\omega) f_{\mathrm{Hxc}}^{\lambda}(\omega) \chi^{\lambda}(\omega) .
$$

Here $\chi^{\mathrm{KS}}(\omega)$ is the noninteracting Kohn-Sham response function and $f_{\mathrm{Hxc}}^{\lambda}(\omega)=\lambda v_{c}+f_{\mathrm{xc}}^{\lambda}(\omega)$ with $v_{c}$ the Coulomb interaction and $f_{\mathrm{xc}}^{\lambda}(\omega)$ the exchange-correlation (xc) kernel. In static density-functional theory (DFT), one needs a rather involved approximation for the xc energy in order to provide a decent description of the ground-state properties of a particular system and no approximation has so far been able to provide accurate results across different binding regimes. In contrast, the ACFDT and Eq. (1) gives a simple framework for calculating correlation energies in terms of the excited states of a noninteracting auxiliary system and is expected to provide a high degree of accuracy with simple approximations for the $\mathrm{xc}$ kernel. In particular, if we use $f_{\mathrm{xc}}=0$ we obtain the random phase approximation (RPA) [4], which has been shown to give an accurate account of dispersive interactions, static correlation, and weak covalent bonds, and is presently considered state of the art in $a b$ initio electronic structure theory involving solid state systems [5-13]. Nevertheless, the RPA suffers from large selfcorrelation errors and predicts too weak binding of solids and molecules, which have severely limited the universal applicability of the method. Furthermore, extending the RPA with semilocal approximations for the xc kernel has been shown to fail dramatically, and progress in "beyond RPA" methods within the framework of TDDFT has so far been rather limited [14-21]. A somewhat orthogonal approach for improving RPA ground-state energies is based on eliminating the RPA self-correlation energy within manybody perturbation theory and is referred to as second-order screened exchange (SOSEX). The SOSEX correlation energy vanishes by construction for any one-electron system and has been shown to improve the accuracy of covalent bonds slightly, while it completely deteriorates the good description of static correlation and barrier heights in the RPA [7,22]. In addition, SOSEX scales as $N^{5}$ with system size and therefore quickly becomes much more computationally demanding than the RPA, which scales as $N^{4}$.

In this Letter, we apply a renormalization scheme that solves the divergence problem associated with semilocal adiabatic TDDFT in the ACDFT. We have implemented the xc kernels for the two most commonly used xc approximations (LDA and PBE) [23] within this renormalization scheme and show that the results obtained with adiabatic renormalized versions are superior to the RPA in all cases. In particular, with the renormalized adiabatic PBE we 
obtain a fourfold improvement of binding energies of molecules and solids, while it maintains the $N^{4}$ scaling of the RPA.

The construction of the renormalized kernel is motivated by the observation that the Fourier transformed correlation hole in the homogeneous electron gas (HEG) is closely reproduced by the exchange part of the Adiabatic Local Density Approximation (Adiabatic LDA) for wave vectors $q<2 k_{F}$. For larger $q$ the exact hole is essentially zero. In contrast, while the ALDA hole vanishes exactly for $q=2 k_{F}$, it attains a finite value for larger $q$ and decays to zero much too slowly. In fact, this long tail produces a divergence of the correlation hole at $r=0$. For $q=2 k_{F}$ the ALDA correlation hole vanishes, since $f_{\mathrm{Hxc}}^{\mathrm{ALDA}}\left(2 k_{F}\right)=4 \pi /\left(2 k_{F}\right)^{2}+f_{x}^{\mathrm{ALDA}}[n]=0$, and it is tempting to define a new renormalized kernel as $f_{\mathrm{Hxc}}^{\mathrm{rALDA}}(q)=\theta\left(2 k_{F}-q\right) f_{\mathrm{Hxc}}^{\mathrm{ALDA}}(q)$ (note that in this work we only consider the exchange part of the LDA and PBE kernels). Alternatively, one can generalize the LDA energy functional to include nonlocal exchange-correlation effects by replacing the local density $n(\mathbf{r})$ by an averaged quantity $n^{*}(\mathbf{r})=\int \phi\left(\mathbf{r}-\mathbf{r}^{\prime}\right) n\left(\mathbf{r}^{\prime}\right) d \mathbf{r}^{\prime}$. This substitution will not alter the ground-state energy or potential of the homogeneous electron gas, but it will introduce nonlocality into the kernel. In fact, it seems physically reasonable that the exchange-correlation energy density should include contributions from the density in the vicinity of the xc hole, which will be the case if $\phi$ has a width similar to the $\mathrm{xc}$ hole. This is accomplished by choosing $\phi$ as the Fourier transform of the step function $\theta\left(2 k_{F}-q\right)$ and we obtain the renormalized kernel as the adiabatic kernel derived from the generalized LDA energy functional. In the Supplemental Material [24] we provide more details on this derivation.

In Refs. [25,26] we investigated this renormalized ALDA (rALDA) kernel in detail and we refer to those papers for more details. The idea is readily generalized to any adiabatic semilocal kernel, $A X$, and we obtain the following expression for the renormalized kernel in real space:

$$
\begin{aligned}
f_{\mathrm{xc}}^{r A X}[n](r)= & \frac{f_{\mathrm{xc}}^{A X}[n]}{2 \pi^{2} r^{3}}\left[\sin \left(q_{c}[n] r\right)-q_{c}[n] r \cos \left(q_{c}[n] r\right)\right], \\
& -\frac{1}{r}\left[1-\frac{2}{\pi} \int_{0}^{q_{c}[n] r} \frac{\sin x}{x} d x\right],
\end{aligned}
$$

where

$$
q_{c}[n]=\sqrt{\frac{-4 \pi}{f_{\mathrm{xc}}^{A X}[n]}} .
$$

Here $q_{c}[n]$ is the cutoff wave vector where the Fourier transform of the correlation hole of the HEG obtained with $f_{\mathrm{xc}}^{A X}$ becomes zero (for $X=\mathrm{LDA}$ the cutoff becomes $q_{c}=2 k_{F}$ ). To generalize the construction to inhomogeneous systems we apply the substitutions

$$
\begin{gathered}
r \rightarrow\left|\mathbf{r}-\mathbf{r}^{\prime}\right|, \\
n \rightarrow \frac{n(\mathbf{r})+n\left(\mathbf{r}^{\prime}\right)}{2}, \\
\nabla n \rightarrow \frac{\nabla_{\mathbf{r}} n(\mathbf{r})+\nabla_{\mathbf{r}^{\prime}} n\left(\mathbf{r}^{\prime}\right)}{2} .
\end{gathered}
$$

Thus both $f_{\mathrm{xc}}^{r A X}$ and $q_{c}$ become functions of $\mathbf{r}$ and $\mathbf{r}^{\prime}$. Importantly, the delta function of the original adiabatic kernel acquires a finite (density-dependent) width, which ensures a finite value of the correlation hole for $\mathbf{r}=\mathbf{r}^{\prime}$ and entails a better description of the short-range correlation effects. We will only discuss non-self-consistent applications of the kernel. This implies that calculated energies will depend on the input density, orbitals, and eigenvalues. This situation is well known from RPA calculations. We note, however, that in contrast to the RPA where no obvious starting point exists, it is natural to base an $r A X$ calculation on a DFT- $X$ calculation. We regard this is as a fundamental merit of the method, since it eliminates the arbitrary choice of input orbitals in non-self-consistent RPA calculations.

We have previously shown that the class of kernels (2) significantly improves the correlation energies of the homogeneous electron gas. Furthermore, using the exchange part of the rALDA kernel reduces the correlation energy of a hydrogen atom to $-0.1 \mathrm{eV}$. This is already a major improvement compared to the RPA which gives $E_{c}=-0.6 \mathrm{eV}$, but the value still comprises a significant self-correlation error. However, inclusion of gradient corrections in the form of PBE exchange reduces the correlation energy to less than $1 \mathrm{meV}$ (less than the resolution of the implementation). In the following we will refer to this kernel as renormalized Adiabatic PBE (rAPBE) and demonstrate the superiority of the resulting kernel over the RPA and other "beyond RPA" methods [27]. The method has been implemented in a plane wave basis in the electronic structure code GPAW $[9,26,28,29]$, which uses the projector augmented wave method [30]. We refer to the Supplemental Material [24] for computational details. We note here that the evaluation of RPA correlation energies is dominated by the calculation of $\chi^{0}$, which scales as $N^{4}$. Evaluating the kernel (2) scales as $N^{2}$ and the method therefore scales as the RPA with system size.

Figure 1 shows the deviation from experimental values of molecular atomization energies. The RPA has a wellknown tendency to underbind and performs somewhat worse than PBE. We also observe a significant difference between RPA@LDA and RPA@PBE with RPA@PBE being the more accurate. The renormalized kernels rALDA and rAPBE show a striking improvement compared to both the RPA and PBE with most errors being on the order of 1-3 kcal/mol. In Fig. 2 we compare the mean absolute relative errors of different methods. It is seen that already the rALDA is better than the SOSEX method and approaches the accuracy of r2PT (SOSEX corrected for 


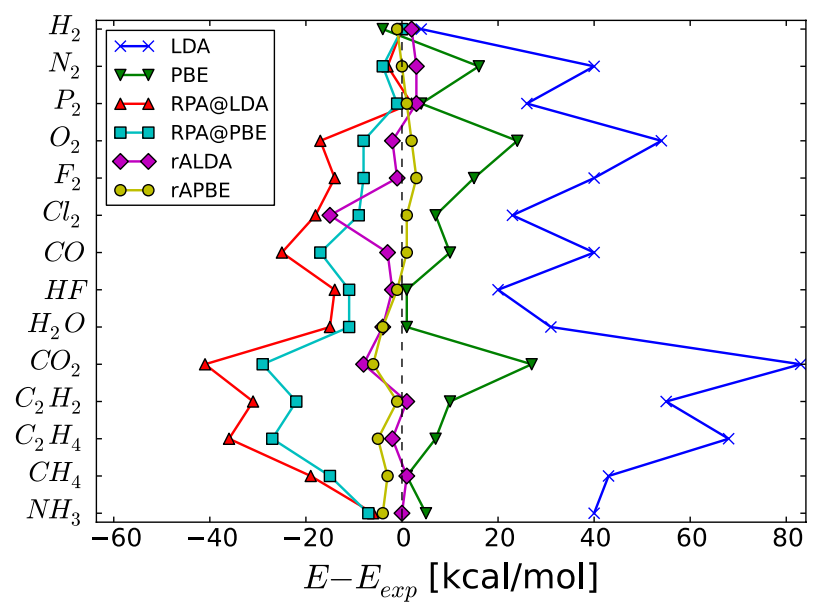

FIG. 1 (color online). Molecular atomization energies evaluated with different methods shown relative to the experimental values. Results are shown with respect to reference values from Ref. [32]. The numbers are tabulated in the Supplemental Material [24].

single excitations [31]) and the hybrid functional PBE0. However, the gradient-corrected rAPBE reduces the error of the rALDA by a factor of 2 and outperforms both PBE0 and rPT2. Since the RPA already performs very well for geometry optimization, we have not performed a detailed comparison of bond lengths with the renormalized kernels. We have only considered the $F_{2}$ and $N_{2}$ molecules where we find deviations from the experimental bond lengths of $1.6 \%(2.4 \%)$ and $0.4 \%(1.2 \%)$ with the rAPBE (RPA), respectively.

In contrast to the case of atomization energies, the RPA is highly accurate for molecular barrier heights where it performs much better than both the SOSEX methods and hybrid functionals [7]. Here we have just tested a single reaction barrier with the $\mathrm{rAPBE}$ functional, namely, $\mathrm{H}+\mathrm{N}_{2} \mathrm{O} \rightarrow \mathrm{OH}+\mathrm{N}_{2}$. For this case we obtain errors of 0.9 (0.4) and $0.2(2.4) \mathrm{kcal} / \mathrm{mol}$ for the forward and backward reactions, respectively, using the rAPBE (RPA) functional. For comparison we obtain errors of 3.2 (5.9) and 12.2 (8.7) using PBE0 (B3LYP). Another important property of the

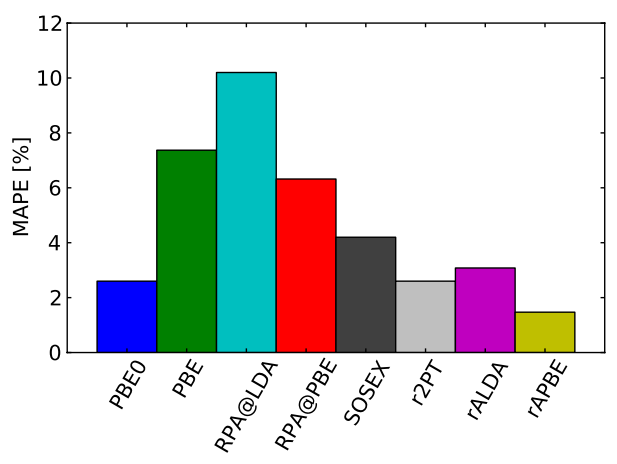

FIG. 2 (color online). Mean absolute percentage deviation of molecular atomization energies. The PBE0, SOSEX, and rP2T values are taken from Ref. [7].

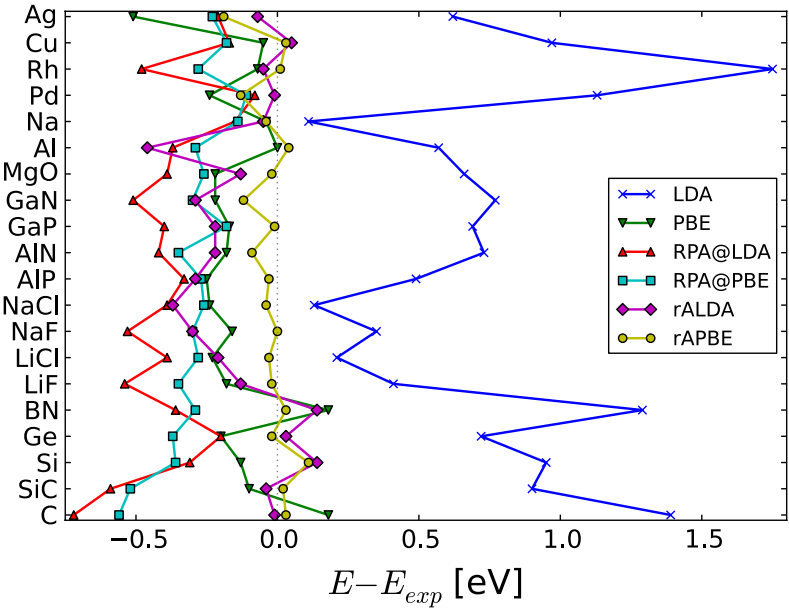

FIG. 3 (color online). Deviation from experimental values of the cohesive energy of solids evaluated with different functionals. The numbers are tabulated in the Supplemental Material [24].

renormalized kernels, is the fact that they describe the dissociation of the $\mathrm{H}_{2}$ molecule correctly in the strict atomic limit [26]. This seems to be a general property of correlation energies from the ACFDT, whenever $\chi$ is derived from an approximation to the irreducible response function such as the Bethe-Salpeter equation or timedependent GW [33].

While the hybrid functionals may be a good choice for a decent accuracy in molecular atomization energies, these methods fail completely for the cohesive energies of solids. The RPA also performs poorly, and the PBE functional seems to be the best choice for this problem. Nevertheless, from Figs. 3 and 4 it is clear that the rALDA functional outperforms the RPA and produces an accuracy similar to PBE. Inclusion of gradient corrections through the rAPBE functional reduces the error by a factor of 4 and is thus much more accurate than any of the other functionals considered. We note in passing that SOSEX has been shown to produce results of similar accuracy to the rAPBE for a small test set of five semiconductors [22], but scales as

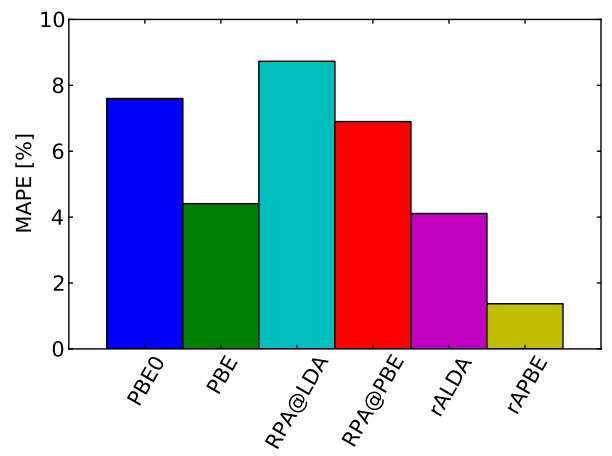

FIG. 4 (color online). Mean absolute percentage deviation of the cohesive energy of solids. The PBE0 results are from Ref. [34]. 
TABLE I. Formation energies of $\mathrm{MgO}$ calculated with different functionals. The experimental value has been corrected for zeropoint energy ${ }^{\mathrm{a}}[11]$.

\begin{tabular}{ccccc}
\hline \hline PBE & HF & RPA & the rAPBE & Expt. \\
\hline 5.34 & 6.04 & 6.12 & 6.22 & 6.26 \\
\hline
\end{tabular}

${ }^{\mathrm{a}}$ All numbers are in $\mathrm{eV}$.

$N^{5}$ with system size and therefore becomes significantly more computationally demanding for solid state systems than the RPA. We have confirmed that the rAPBE functional inherits the good description of lattice constants within the RPA [12]. In particular, we have calculated the lattice constants for bulk $\mathrm{C}, \mathrm{Si}, \mathrm{Na}$, and $\mathrm{Pd}$. The results are provided in the Supplemental Material [24]. The deviations from experimental values corrected for zero point anharmonic effects are $0.4 \%(0.8 \%), 0.6 \%(0.6 \%), 1.8 \%(1.4 \%)$, and $1.8 \%(1.4 \%)$ for the rAPBE (RPA), respectively.

One might speculate that the rAPBE accuracy of atomization energies and cohesive energies of solids is simply due to a better description of the isolated atoms compared to the RPA. Of course, the already much improved correlation energy of the HEG compared to the RPA indicates that this is not the case. As another demonstration of this fact we have calculated the formation energy of $\mathrm{MgO}$ defined as the energy of the reaction $\mathrm{Mg}(\mathrm{s})+1 / 2 \mathrm{O}_{2}(\mathrm{~g}) \rightarrow \mathrm{MgO}(\mathrm{s})$. The result is shown in Table I. As can be seen, the rAPBE reduces the RPA error by $0.1 \mathrm{eV}$ corresponding to a fourfold decrease in relative error. Thus, the renormalized kernel does not only improve the description of isolated atoms relative to the RPA, but gives rise to a universal improvement of correlation energies that manifests itself in any calculated quantity.

One of the great success stories of the RPA, is that it solves the famous "CO puzzle." Most generalized gradient approximations (GGAs) predict the wrong adsorption site for $\mathrm{CO}$ on metal surfaces, whereas the RPA predicts the correct adsorption order [13]. Furthermore, it is possible to choose a semilocal functional that gives the correct adsorption energy, but this will be at the cost of a highly inaccurate metal surface energy. In contrast, the RPA produces an accurate adsorption energy and still yields a reasonable metal surface energy. As shown in Fig. 5, this trend is inherited by the rAPBE functional which produces results very similar to those of the RPA. For comparison we also show the results for various GGAs [13] and van der Waals functionals [35].

Another successful application of the RPA method is the adsorption of graphene on metal surfaces [8,9,36]. Graphene interacts with metals through long-range dispersive and short-range weak covalent interactions. The adsorption geometry and binding energy are determined by a detailed balance between these contributions which are of equal magnitude. Semilocal functionals cannot account for the dispersive interactions and in many cases do not provide

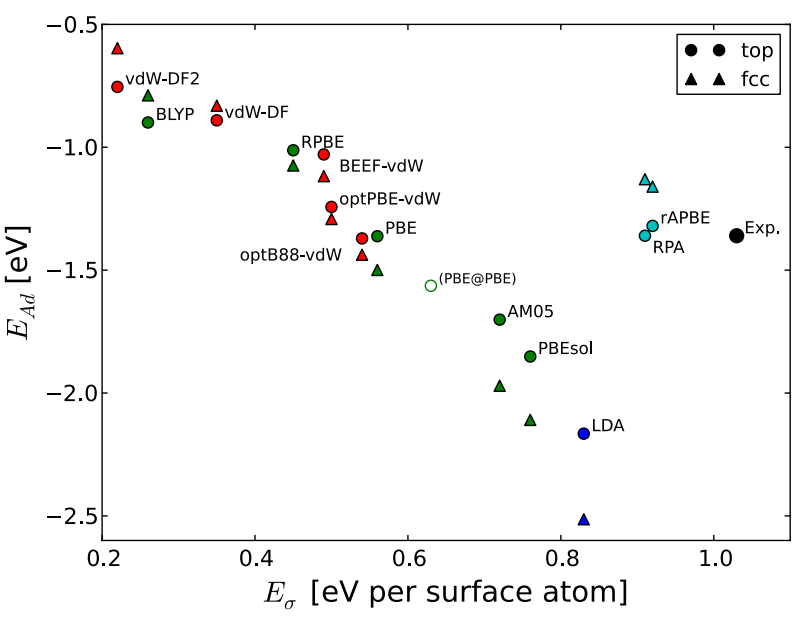

FIG. 5 (color online). Surface energy versus adsorption energy of $\mathrm{CO} / \mathrm{Pt}(111)$ calculated with various GGA functionals (green markers) and van der Waals functionals (red markers). Circles and triangles indicate atop and hollow sites, respectively. All calculations were performed with the experimental lattice constant of Pt and the CO molecule relaxed with PBE. The hollow circle was obtained with a PBE optimized lattice constant.

the required accuracy for weak covalent interactions either. On the other hand, most van der Waals functionals account well for the dispersive interactions at large distances, but fail at shorter distances due to an incorrect description of the exchange interaction $[35,36]$. The RPA predicts metalgraphene binding distances in overall good agreement with experiments. In particular, for the case of $\mathrm{Ni}(111)$ and $\mathrm{Co}(0001)$ the RPA yields two distinct minima around $d=2.2 \AA$ and $d=3.3 \AA$, corresponding to (weak) chemisorption and physisorption, respectively. The chemisorption minimum is slightly deeper in good agreement with experimental findings of $d \approx 2.1 \AA$. However, due to the inaccurate description of covalent bonds in molecules and

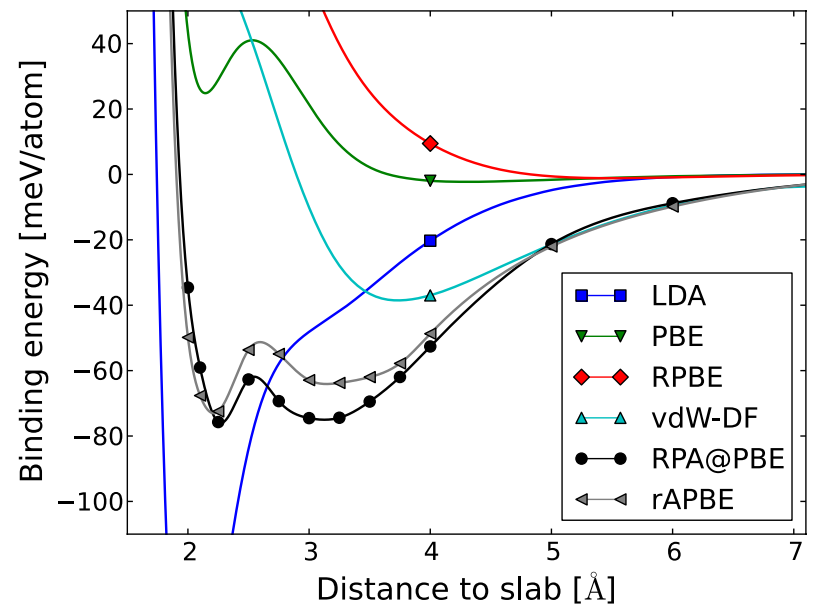

FIG. 6 (color online). Potential energy curve for graphene on $\mathrm{Ni}(111)$ calculated with different methods. The experimental binding distance is $d \approx 2.1 \AA$. 
solids one could question the accuracy of the RPA description of the chemisorption minimum. So far, the RPA has been the best possible method to analyze these systems, since quantum chemistry methods are out of the question for this class of systems. We have calculated the binding energy curve for graphene on $\mathrm{Ni}(111)$ using the rAPBE kernel and compared it to various other methods, see Fig. 6. It is seen that the rAPBE and the RPA produce very similar results. As one could perhaps expect from the general tendency of the RPA to underbind, the rAPBE kernel lowers the chemisorption minimum relative to the physisorption minimum. While this change is in the right direction compared to experiments, it does not change the qualitative picture of the binding obtained from the RPA.

In conclusion, we have shown a way to construct xc kernels within TDDFT that extends the RPA in a natural way and improves its description of short-range correlations while retaining the good description of dispersive interactions and static correlation with similar computational cost. The proposed renormalization procedure can be applied to any known semilocal xc functional and thus defines an entirely new class of adiabatic nonlocal xc kernels, which could pave the way for achieving chemical accuracy in solid state calculations.

The authors acknowledge support from the Danish Council for Independent Research's Sapere Aude Program, Grant No. 11-1051390. The Center for Nanostructured Graphene is sponsored by the Danish National Research Foundation, Project DNRF58.

*tolsen@fysik.dtu.dk

[1] R. J. Bartlett and M. Muslat, Rev. Mod. Phys. 79, 291 (2007).

[2] G. H. Booth, A. Grüneis, G. Kresse, and A. Alavi, Nature (London) 493, 365 (2013)

[3] J. J. Shepherd and A. Grüneis, Phys. Rev. Lett. 110, 226401 (2013).

[4] D. Bohm and D. Pines, Phys. Rev. 82, 625 (1951).

[5] F. Furche, Phys. Rev. B 64, 195120 (2001).

[6] H. Eshuis, J. E. Bates, and F. Furche, Theor. Chem. Acc. 131, 1084 (2012).

[7] X. Ren, P. Rinke, C. Joas, and M. Scheffler, J. Mater. Sci. 47, 7447 (2012).

[8] T. Olsen, J. Yan, J. J. Mortensen, and K. S. Thygesen, Phys. Rev. Lett. 107, 156401 (2011).

[9] T. Olsen and K.S. Thygesen, Phys. Rev. B 87, 075111 (2013)

[10] J. Yan, J. S. Hummelshøj, and J. K. Nørskov, Phys. Rev. B 87, 075207 (2013).
[11] J. Harl and G. Kresse, Phys. Rev. Lett. 103, 056401 (2009).

[12] J. Harl, L. Schimka, and G. Kresse, Phys. Rev. B 81, 115126 (2010).

[13] L. Schimka, J. Harl, A. Stroppa, A. Grüneis, M. Marsman, F. Mittendorfer, and G. Kresse, Nat. Mater. 9, 741 (2010).

[14] M. Lein, E. K. U. Gross, and J. P. Perdew, Phys. Rev. B 61, 13431 (2000).

[15] F. Furche and T. V. Voorhis, J. Chem. Phys. 122, 164106 (2005).

[16] J. F. Dobson and J. Wang, Phys. Rev. B 62, 10038 (2000).

[17] J. Jung, P. Garcia-González, J. F. Dobson, and R. W. Godby, Phys. Rev. B 70, 205107 (2004).

[18] T. Gould, J. Chem. Phys. 137, 111101 (2012).

[19] A. Heßelmann and A. Görling, Mol. Phys. 108, 359 (2010).

[20] A. Heßelmann and A. Görling, Phys. Rev. Lett. 106, 093001 (2011).

[21] D. Lu, J. Chem. Phys. 140, 18A520 (2014).

[22] A. Grüneis, M. Marsman, J. Harl, L. Schimka, and G. Kresse, J. Chem. Phys. 131, 154115 (2009).

[23] J. P. Perdew, K. Burke, and M. Ernzerhof, Phys. Rev. Lett. 77, 3865 (1996).

[24] See Supplemental Material at http://link.aps.org/ supplemental/10.1103/PhysRevLett.112.203001 for an alternative derivation of the renormalized kernels, calculational details, the data used to generate Figs. 1-4, and optimized rAPBE geometries of a few solids and molecules.

[25] T. Olsen and K. S. Thygesen, Phys. Rev. B 86, 081103(R) (2012).

[26] T. Olsen and K. S. Thygesen, Phys. Rev. B 88, 115131 (2013).

[27] For simplicity, we have neglected the variation in the potential with respect to the density gradient and thus use $f_{x}^{\mathrm{APBE}}[n]=\left.\left(\partial v_{x}^{\mathrm{PBE}}(n) / \partial n\right)\right|_{n=n(\mathbf{r})}$ to construct the $\mathrm{rAPBE}$ kernel.

[28] J. Enkovaara et al., J. Phys. Condens. Matter 22, 253202 (2010).

[29] J. Yan, J. J. Mortensen, K. W. Jacobsen, and K. S. Thygesen, Phys. Rev. B 83, 245122 (2011).

[30] P. E. Blöchl, Phys. Rev. B 50, 17953 (1994).

[31] X. Ren, A. Tkatchenko, P. Rinke, and M. Scheffler, Phys. Rev. Lett. 106, 153003 (2011).

[32] A. Karton, E. Rabinovich, J. M. L. Martin, and B. Ruscic, J. Chem. Phys. 125, 144108 (2006).

[33] T. Olsen and K. S. Thygesen, J. Chem. Phys. 140, 164116 (2014).

[34] J. Paier, M. Marsman, K. Hummer, G. Kresse, I. C. Gerber, and J. G. Angyan, J. Chem. Phys. 124, 154709 (2006).

[35] J. Wellendorff, K. T. Lundgaard, A. Møgelhøj, V. Petzold, D. D. Landis, J. K. Nørskov, T. Bligaard, and K. W. Jacobsen, Phys. Rev. B 85, 235149 (2012).

[36] F. Mittendorfer, A. Garhofer, J. Redinger, J. Klimes, J. Harl, and G. Kresse, Phys. Rev. B 84, 201401 (2011). 